\title{
Test de Findrisk y predicción de diabetes mellitus tipo dos, en alumnos de la Escuela de Medicina Humana de la Universidad Nacional José Faustino Sánchez Carrión - 2017
}

Findrisk test and prediction of diabetes mellitus type two, in students of the School of Human Medicine of the José Faustino Sánchez Carrión National University - 2017

Soledad Dionisia LLañez Bustamante ${ }^{1}$ Isaúl Mauricio Alor Herbozo², Geraldina Fortunata Paredes Bottoni ${ }^{3}$, Vásquez Estela Darío Estanislao ${ }^{3}$, Mirella Heidi Alor LLañez ${ }^{3}$

\section{RESUMEN}

Objetivo: Determinar el valor del Test de Findrisk y la predicción de diabetes mellitus tipo dos, en alumnos de la Escuela de Medicina Humana en la Universidad Nacional José Faustino Sánchez Carrión -2017. Métodos: Investigación básica, descriptiva, correlacional de corte transversal, que permitió establecer la relación entre las dos variables. La muestra estuvo conformada por 60 estudiantes entre 16 y 30 años de edad de ambos sexos de una población de 320 . Para la recolección de datos se aplicó un cuestionario. Resultados: En relación al Índice de masa corporal (IMC), del total de los estudiantes del estudio $35 \%$ se encontró en estado normal; $41,5 \%$ tuvo sobrepeso y $23,3 \%$ obeso. Para el perímetro de cintura $63,3 \%$ estuvo dentro del rango normal; $58,3 \%$ realiza actividad física al menos 30 minutos al día en sus tiempos libres; $36,7 \%$ se obtuvo para el parámetro consume verduras todos los días, y el mismo porcentaje para el consumo de 3 veces por semana. Ninguno de ellos tuvo valores altos de hipertensión arterial y para los antecedentes de diabetes en sus familiares directos y parientes se obtuvo respuestas positivas de $11,6 \%$ y $26,7 \%$ respectivamente y un $61,7 \%$ respondió que no tuvieron antecedentes familiares con diabetes. Conclusion: PSegún el Test de Findrisk; $58,3 \%$ de alumnos de la Escuela de Medicina Humana en la Universidad Nacional José Faustino Sánchez Carrión - 2017 presentó, riesgo bajo, el 15,0\% riesgo moderado, el 13,3\% riesgo muy alto y el 10,0\% riesgo alto, de padecer diabetes mellitus tipo dos de aquí a 10 años.

Palabras clave: Diabetes mellitus tipo dos, Test de Findrisk, Perímetro de Cintura, Índice de masa corporal, Glucemia.

\section{ABSTRACT}

Objective: To determine the value of the Findrisk test and the prediction of type two diabetes mellitus in students of the School of Human Medicine at the José Faustino Sánchez Carrión National University 2017. Methods: Basic, descriptive, cross-sectional correlation research, which allowed establishing the relationship between the two variables. The sample consisted of 60 students between 16 and 30 years of age of both sexes from a population of 320. For the data collection a questionnaire was applied. Results: In relation to the Body Mass Index (BMI), of the total of the students in the study, $35 \%$ were in a normal state; $41.5 \%$ were overweight and $23.3 \%$ obese. For the waist circumference $63.3 \%$ was within the normal range; $53.3 \%$ perform physical activity at least 30 minutes a day in their free time; $36.7 \%$ was obtained for the parameter consumed vegetables every day, and the same percentage for the consumption of 3 times per week. None of them had high values of arterial hypertension and for the history of diabetes in their direct relatives and relatives positive responses were obtained of $11.6 \%$ and $26.7 \%$ respectively and $61.7 \%$ answered that they did not have relatives with diabetes. Conclusion: FAccording to the Findrisk test; $58.3 \%$ of students of the School of Human Medicine at the José Faustino Sánchez Carrión National University - 2017 presented, low risk, 15.0\% moderate risk, 13.3\% very high risk and $10.0 \%$ risk high, to suffer type two diabetes mellitus in 10 years.

Keywords: YaDiabetes mellitus type two, Findrisk test, Waist circumference, Body mass index, Glycemia. 


\section{INTRODUCCIÓN}

La Organización Mundial (OMS), calcula que en el mundo hay más de 170 millones de personas con diabetes, cifra que podría duplicarse en el 2030. La importancia sanitaria de la diabetes deriva de su magnitud, ya que es la enfermedad endocrina más frecuente; de su trascendencia, asociada a una mayor morbimortalidad; de su coste, individual y social; de sus complicaciones y de sus posibilidades de control mediante medidas de prevención de la enfermedad. (Sagarra, Costa, Cabré, Solà-Morales, y Barrio (2014).

Las tasas de diabetes tipo 2 han aumentado notablemente desde 1960, en paralelo con la obesidad. Para 2010 había aproximadamente 285 millones de personas diagnosticadas con la enfermedad en comparación con alrededor de 30 millones en 1985. La diabetes tipo 2 es típicamente una enfermedad crónica asociada con una disminución de la esperanza de vida de diez años. Las complicaciones a largo plazo de la hiperglucemia pueden incluir enfermedades del corazón, derrames cerebrales, retinopatía diabética (afecta la vista), insuficiencia renal que puede requerir diálisis y mala circulación en las extremidades que conduce a amputaciones. La cetoacidosis, una complicación aguda y característica de la diabetes tipo 1, es poco común, sin embargo puede ocurrir un coma hiperosmolar hiperglucémico. (OMS 2016 Diabetes Centro de prensa Nota descriptiva Noviembre de 2016.

El Ministerio de salud ha establecido de nuestro país, en los lineamientos de política del sector 2007-2020, la vigilancia, prevención y control de las enfermedades transmisibles y no transmisibles, el control de enfermedades crónica degenerativas, priorizando diabetes mellitus (DM), hipertensión arterial, enfermedad isquémica del miocardio y accidente cerebrovascular, como una prioridad. El Sistema de vigilancia de diabetes en servicios de salud se desarrolla desde el año 2010 como un sistema piloto en algunos hospitales de Lima y de las regiones del país, el mismo que se encuentra en proceso de evaluación (OMS, 2016).
Desde el inicio de la vigilancia epidemiológica de diabetes al I semestre de 2013 se han registrado 5001 casos de diabetes, en 16 Hospitales (seis de ellos en Lima) y en una clínica privada de Lima. Entre enero y junio de 2013, se han registrado 928 casos, esto representa el $18,6 \%$ de los casos registrados desde el inicio del piloto y el $24,2 \%$ de los casos registrados el año 2012. (Revilla, 2013).

Los procesos mediante los cuales las instituciones logran captar personas con riesgo de diabetes tipo 2 no son lo suficientemente eficaces generando que cada año espontáneamente lleguen a los servicios personas adultas con complicaciones de tipo crónico relacionadas con la diabetes. El primer paso hacia la prevención, es la detección precoz de estas poblaciones, mediante procedimientos de tamizaje 0 "Screening" ocasional, los cuales permiten abordar tempranamente el problema y establecer estrategias de prevención, dirigidas a poblaciones específicas. Sin embargo esto no es suficiente; para prevenir la aparición de la diabetes, se deben además identificar aquellos individuos que tengan el riesgo de padecerla. Entre las diferentes formas de prevención se recomienda utilizar el Test de FINDRISK, que es válido para la detección precoz. (ADA, 2015).

En el presente estudio se ha determinado la predicción de diabetes mellitus tipo dos, en alumnos de la Escuela de Medicina Humana en la Universidad Nacional José Faustino Sánchez Carrión - 2016 mediante el uso del Test de FINDRISK.

\section{MATERIAL Y MÉTODOS}

El tipo de investigación fue básica, descriptiva, correlacional y corte transversal, y permitió establecer la relación entre las dos variables. La Población estuvo conformada por 320 alumnos y de ellos una muestra de 60 alumnos mayores de 16 años y menores de 30 años varones y damas de la Escuela de Medicina de La Universidad Nacional José Faustino Sánchez Carrión.

Se aplicó cuestionario para la Variable 2: Riesgo de padecer diabetes mellitus tipo dos y 
para la Variable 1: Valor del Test de Findrisk; es un instrumento sencillo, válido y útil para determinar el riesgo de diabetes que posee las dimensiones, Antecedentes, Hábitos Alimentarios, Actividad física, Hiperglucemia y Deficiencia de insulina.
Se empleó programas estadísticos: SPSS, y a través de Tablas y Figuras se analizaron e interpretaron los resultados para obtener las conclusiones de la investigación.

\section{RESULTADOS}

Tabla 1. Edad del paciente

\begin{tabular}{ccccc}
\hline & \multicolumn{3}{c}{ Hombre } & \multicolumn{2}{c}{ Mujer } \\
Categorías & Frecuencia & $\%$ & Frecuencia & $\%$ \\
$17-20$ años & 15 & $50,0 \%$ & 22 & $73,3 \%$ \\
$21-25$ años & 9 & $30,0 \%$ & 8 & $26,7 \%$ \\
$26-30$ años & 6 & $20,0 \%$ & 0 & $0,0 \%$ \\
Total & 30 & $100,0 \%$ & 30 & $100,0 \%$ \\
\hline
\end{tabular}

Fuente: Elaboración propia

En la Tabla 1 se puede apreciar que el rango de edad comprendida fue de 17 a 30 años, predominando en ambos grupos tanto los hombres como las mujeres la edad entre 17 20 años, ocupando un alto porcentaje con un $73,3 \%$ las estudiantes mujeres y estudiantes hombres en un $50,0 \%$ de la Escuela de
Medicina de la Universidad Nacional José Faustino Sánchez Carrión.

Le seguían con un $30 \%$ y $26,7 \%$ respectivamente las categorías de edad entre 21 - 25 años y con $20 \%$ las categorías de edad comprendida entre 26 - 30 años.

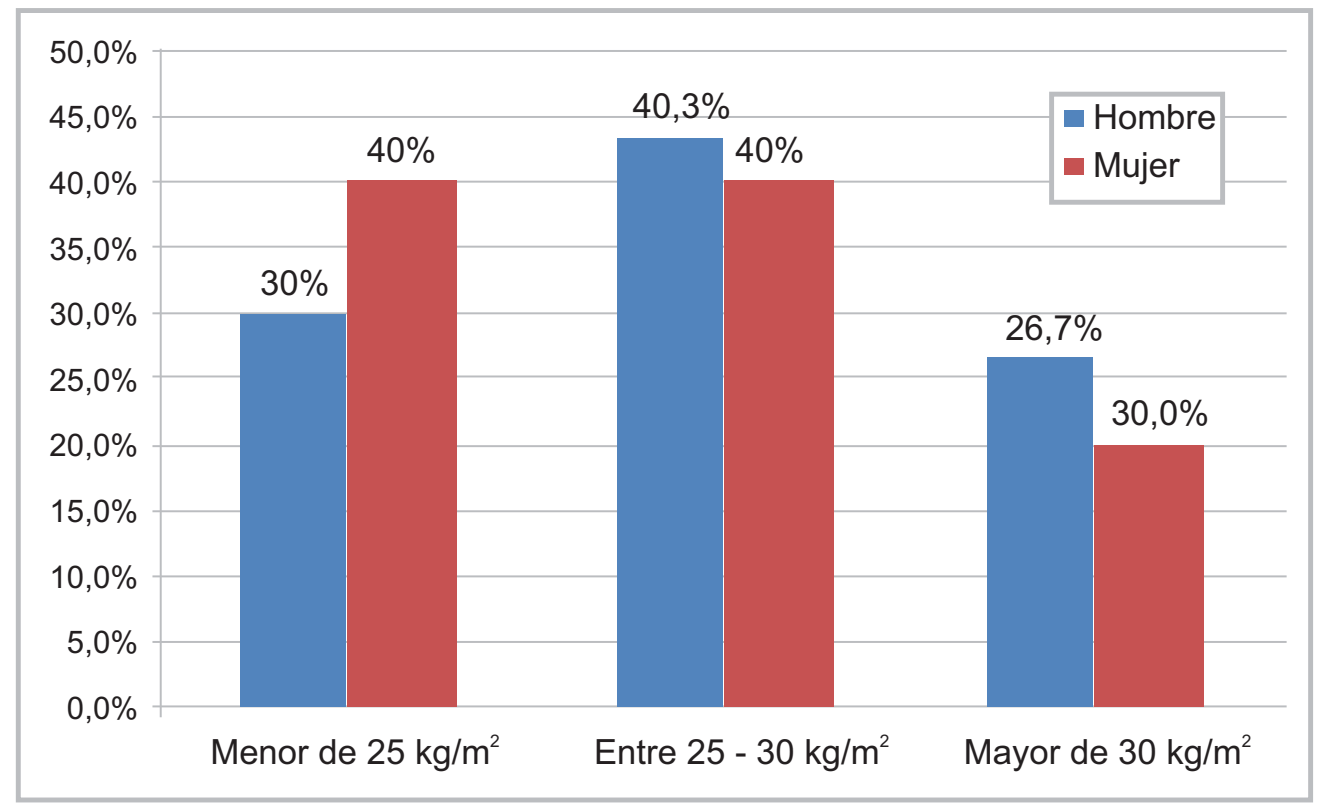

Figura 1. Indice de masa corporal

En la mayoría de los estudiantes diagnosticados de la Escuela de Medicina de la Universidad Nacional José Faustino Sánchez Carrión, presentan un índice de masa corporal entre $25-30 \mathrm{~kg} / \mathrm{m} 2$, se encontró que los estudiantes hombres tenían mayor porcentaje $43,3 \%$ que las mujeres $40,0 \%$. 
Tabla 2. Perímetro de cintura medido por debajo de las costillas (a nivel ombligo)

\begin{tabular}{ccccc}
\hline & \multicolumn{3}{c}{ Hombre } & \multicolumn{2}{c}{ Mujer } \\
\cline { 2 - 5 } Categorías & Frecuencia & $\%$ & 18 & $60,0 \%$ \\
Menos de $80 \mathrm{~cm}$ & 17 & $56,7 \%$ & 12 & $40,0 \%$ \\
Entre $80-88 \mathrm{~cm}$ & 11 & $36,7 \%$ & 0 & $0,0 \%$ \\
Más de $88 \mathrm{~cm}$ & 2 & $6,7 \%$ & $\mathbf{3 0}$ & $\mathbf{1 0 0 , 0} \%$ \\
Total & $\mathbf{3 0}$ & $\mathbf{1 0 0 , 0 \%}$ &
\end{tabular}

Fuente: Elaboración propia

De los estudiantes diagnosticados por perímetro de cintura medido por debajo de las costillas menores a $80 \mathrm{~cm}$, se observó mayor porcentaje en estudiantes mujeres $60 \%$ y un
$56,7 \%$ en hombres estudiantes de la Escuela de Medicina de la Universidad Nacional José Faustino Sánchez Carrión (Tabla 2).inicial y en proceso de concentración.

Tabla 3. ¿Realiza habitualmente al menos 30 minutos de actividad física, diaria en la universidad, trabajo y/o en el tiempo libre?

\begin{tabular}{ccccc}
\hline & & Hombre & \multicolumn{2}{c}{ Mujer } \\
\cline { 2 - 5 } Categorias & Frecuencia & $\%$ & Frecuencia & $\%$ \\
$\mathrm{Si}$ & 15 & $50,0 \%$ & 10 & $33,3 \%$ \\
No & 15 & $50,0 \%$ & 20 & $66,7 \%$ \\
Total & 30 & $\mathbf{1 0 0 , 0} \%$ & $\mathbf{3 0}$ & $\mathbf{1 0 0 , 0 \%}$
\end{tabular}

Fuente: Elaboración propia

Un $66,7 \%$ de mujeres y un $50,0 \%$ de hombres estudiantes de la Escuela de Medicina de la Universidad Nacional José Faustino Sánchez Carrión afirman que si realizan habitualmente al menos 30 minutos de actividad física, diaria en la universidad, trabajo y/o en el tiempo libre (Tabla 3).

Tabla 4. ¿Con qué frecuencia comen verduras o frutas?

\begin{tabular}{|c|c|c|c|c|}
\hline \multirow[b]{2}{*}{ Categorias } & \multicolumn{2}{|c|}{ Hombre } & \multicolumn{2}{|c|}{ Mujer } \\
\hline & Frecuencia & $\%$ & Frecuencia & $\%$ \\
\hline Todos los días & 4 & $13,3 \%$ & 11 & $36,7 \%$ \\
\hline 3 veces a la semana & 20 & $66,7 \%$ & 11 & $36,7 \%$ \\
\hline Una vez a la semana & 6 & $20,0 \%$ & 8 & $26,7 \%$ \\
\hline Consumo verduras o frutas cada 15 días & 0 & $0,0 \%$ & 0 & $0,0 \%$ \\
\hline No consumo verduras ni frutas & 0 & $0,0 \%$ & 0 & $0,0 \%$ \\
\hline Total & 30 & $100,0 \%$ & 30 & $100,0 \%$ \\
\hline
\end{tabular}

Fuente: Elaboración propia

En la Tabla 4 se observa que el grupo que más consumió verduras o frutas en un $66,7 \%$ son hombres estudiantes, seguido de un $36,7 \%$ de mujeres estudiantes de la Escuela de Medicina de la Universidad Nacional José
Faustino Sánchez Carrión, quienes consumen verduras o frutas 3 veces por semana; en cambio el $26,7 \%$ de mujeres y el $20 \%$ de hombres estudiantes consumen verduras y fruta una vez por semana.

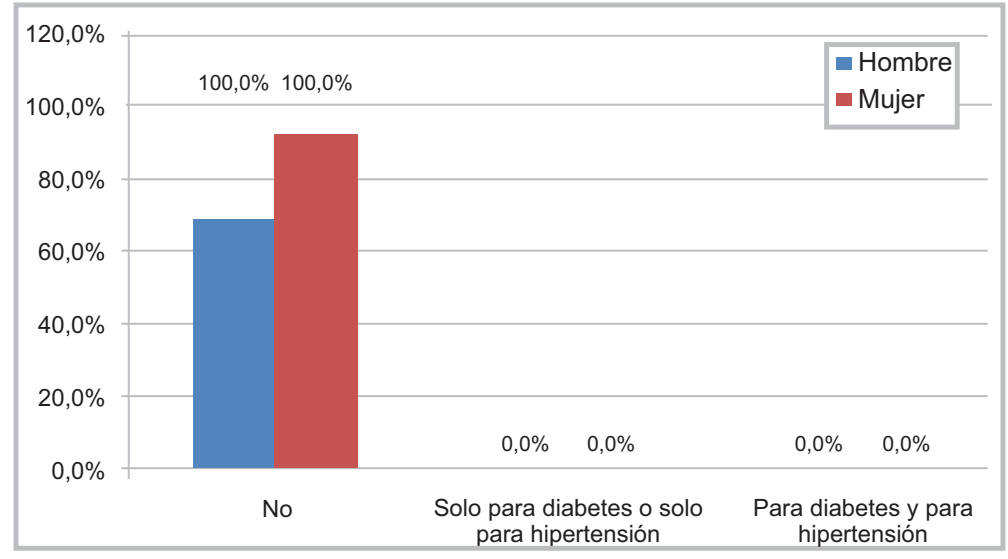

Figura 2. ¿Toma medicación para la hipertensión arterial o para la diabetes regularmente? 
En la Figura 2 el $100,0 \%$ de estudiantes de ambos sexos de la Escuela de Medicina de la Universidad Nacional José Faustino Sánchez
Carrión no toman medicación para la hipertensión arterial o para la diabetes regularmente.

Tabla 5. ¿Le han encontrado alguna vez valores de glucosa altos(Ej. En un control médico, durante una enfermedad, durante el embarazo)?

\begin{tabular}{ccccc}
\hline & \multicolumn{3}{c}{ Hombre } & \multicolumn{2}{c}{ Mujer } \\
\cline { 2 - 4 } Categorias & Frecuencia & $\%$ & Frecuencia & $\%$ \\
No & 24 & $80,0 \%$ & 28 & $93,3 \%$ \\
Si & 6 & $20,0 \%$ & 2 & $6,7 \%$ \\
Total & 30 & $100,0 \%$ & 30 & $100,0 \%$
\end{tabular}

Fuente: Elaboración propia

Al $93,3 \%$ de mujeres y $80,0 \%$ de hombres estudiantes de la Escuela de Medicina de la Universidad Nacional José Faustino Sánchez Carrión no le han encontrado alguna vez valores de glucosa altos. Un $20 \%$ de hombres y $6,7 \%$ de mujeres si le han encontrado alguna vez valores de glucosa altos (Tabla 5).

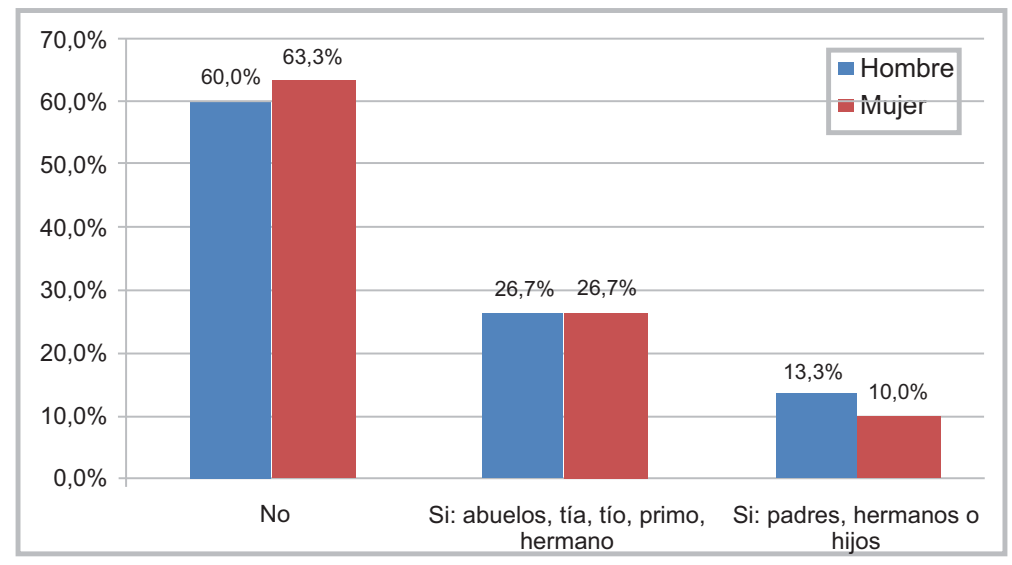

Figura 3. ¿Se le ha diagnosticado diabetes (tipo 1 o tipo 2) a alguno de sus familiares allegados u otros parientes?

En la Figura 3 muestra que el $63,3 \%$ de mujeres y un $60 \%$ de hombres estudiantes de la Escuela de Medicina de la Universidad Nacional José Faustino Sánchez Carrión comunicó que no se le ha diagnosticado diabetes (tipo 1 o tipo 2) a alguno de sus familiares allegados $u$ otros parientes; un $26,7 \%$ en ambos sexos comunicó que si presentaron antecedentes de segundo grado (abuelos, tías, tíos, primos hermanos) y un $13,3 \%$ hombres, $10 \%$ mujeres si presentaron de primer grado (Padres, hermanos o hijos).

Hipótesis Alternativa Ha: El valor del Test de Findrisk predice un riesgo bajo de diabetes mellitus tipo dos, en alumnos de la Escuela de Medicina Humana en la Universidad Nacional José Faustino Sánchez Carrión -2016.

Tabla 6. Test de Findrisk

\begin{tabular}{|c|c|c|c|c|c|}
\hline & & Frecuencia & Porcentaje & Porcentaje válido & $\begin{array}{l}\text { Porcentaje } \\
\text { acumulado }\end{array}$ \\
\hline \multirow{6}{*}{ Válidos } & Riesgo alto & 6 & 10,0 & 10,0 & 10,0 \\
\hline & Riesgo bajo & 35 & 58,3 & 58,3 & 68,3 \\
\hline & $\begin{array}{l}\text { Riesgo ligeramente } \\
\text { aumentado }\end{array}$ & 2 & 3,3 & 3,3 & 71,7 \\
\hline & Riesgo moderado & 9 & 15,0 & 15,0 & 86,7 \\
\hline & Riesgo muy alto & 8 & 13,3 & 13,3 & 100,0 \\
\hline & Total & 60 & 100,0 & 100,0 & \\
\hline
\end{tabular}

Como se muestra en la Tabla 6 el Test de Findrisk muestra que un $58,3 \%$ de alumnos de la Escuela de Medicina Humana en la Universidad Nacional Josè Faustino Sánchez
Carrión - 2016 presentan un riesgo bajo, un $15,0 \%$ muestran un riesgo moderado, un $13,3 \%$ un riesgo muy alto y un $10,0 \%$ exhiben un riesgo alto. 


\section{DISCUSIÓN}

Los resultados mostrados a partir de la Tabla 1 , indican que el mayor porcentaje eran mujeres $73,3 \%$ distribuida entre las edades de 17 a 20 años y solo en un $20 \%$ de hombres se hallan los de 26 a 30 años. En relación a los valores que arrojan la medida del IMC (Figura 1) en la muestra estudiada se obtuvo $35 \%$ en estado normal; $41,5 \%$ de sobrepeso y $23,3 \%$ de obeso. Para el perímetro de cintura $63,3 \%$ normal; el $58,3 \%$ realiza actividad física al menos treinta minutos por día o en su tiempo libre, es satisfactorio encontrar que un 36,7\% de mujeres consume verduras y /o frutas 3 veces por semana y un porcentaje igual lo hace todos los días. Ninguno de ellos tuvo valores altos de hipertensión arterial. Y para los antecedentes de diabetes en sus familiares directos y parientes se obtuvo respuestas positivas de $11,6 \%$ y $26,7 \%$ respectivamente que si presentaron antecedentes y un $61,7 \%$ respondió que no tuvieron antecedentes familiares con diabetes. Los valores del Test de Findrisk indicaron que el $58,3 \%$ de alumnos de la Escuela de Medicina Humana en la Universidad Nacional José Faustino Sánchez Carrión - 2016 presentan un riesgo bajo, un $15,0 \%$ muestran un riesgo moderado, un $13,3 \%$ un riesgo muy alto y un $10,0 \%$ exhiben un riesgo alto.

Todos estos valores son menores a los hallados por Palacios, Durán y Obregón (2012), por lo que se asume que la edad de las personas guarda directa relación con el incremento de muchos valores de los parámetros considerados por el Test de Findrisk .

Paredes et al. (2014), Aplicó el Test Findrisk a 404 personas no diabéticas mayores de 20 años obtuvieron resultados semejantes a los de nuestra muestra siendo los parámetros de mayor coincidencia el sobrepeso $(41,34 \%)$, obesidad $(19,80 \%)$ lo cual conllevo a su muestra a un riesgo alto de presentar diabetes después de 10 años del estudio, los valores son muy parecidos, los alumnos tienen un $10 \%$ y en el estudio de Paredes es un $0,99 \%$ en riesgo muy alto y un $13,8 \%$ son hipertensos. Rodríguez (2013), aplicó el Test de Findrisk en una muestra de 256 personas entre 20 a 40 años de edad, y sus resultados comparando con los alumnos de Medicina Humana, presentaron en todos los parámetros que usa el Test de Findrisk riesgos significativamente más elevado que el estudio de Rodríguez.

Flores y Marín (2013) identifican factores de riesgo para diabetes mellitus tipo 2 en una población de 134 adultos: encontrando valores muy altos en comparación con los alumnos de Medicina, y el $89,6 \%$ de la población existiendo siendo el parámetro más representativo en ese trabajo que la población adulta, no realiza actividad física.

Según el Test de Findrisk aplicado a los alumnos de la Escuela de Medicina Humana en la Universidad Nacional José Faustino Sánchez Carrión - 2016, se concluye que el $58,3 \%$ de la población encuestada tienen un riesgo bajo de desarrollar Diabetes mellitus tipo dos, el $15,0 \%$ muestran un riesgo moderado el $10,0 \%$ y el $13,3 \%$ un riesgo muy alto de padecer diabetes mellitus tipo dos, de aquí a 10 años.

\section{REFERENCIAS BIBLIOGRÁFICAS}

American Diabetes Association. ADA (2015). Classification and diagnosis of diabetes. Diabetes care, 38(Supplement 1), S8S16.

Flores Gonzales, E. K. \& Marin Ramírez, C.K. (2013). Factores de riesgo para diabetes mellitus tipo II en adultos del sector" Atumpampa" distrito de Tarapoto 2012.

OMS (2016). Informe mundial sobre la Diabetes: Resumen de Orientación. Ginebra, Organización Mundial de la Salud.

Palacios, A., Durán, M., \& Obregón, O. (2012). Factores de riesgo para el desarrollo de diabetes tipo 2 y síndrome metabólico. Revista Venezolana de Endocrinología y Metabolismo, 10, 34-40.

Paredes, N., Materano, M., Ojeda, A., López, J., López, A., Rosales, J. \& Chacón-Lozsán, F. (2014). Aplicación del test Findrisk para cálculo del riesgo de padecer diabetes mellitus tipo 2. Medicina Interna, 30(1). 
Ponce Pardo, K. K. \& Benites Paredes, K. B. (2015). Factores de riesgo de diabetes mellitus tipo 2 en el personal administrativo de la universidad privada Antenor Orrego. Trujillo, 2014. Tesis para obtener el título profesional de licenciada en enfermería. Universidad Privada Antenor Orrego, Trujillo.

Revilla, L. (2013). Situación de la vigilancia de diabetes en el Perú, al I semestre de 2013. Bol epidemiol, 22(39), 4.

Rodríguez, A. (2013). Riesgo de Diabetes Mellitus Tipo 2 en la población entre 20 a 40 años del Canton Catamayo en el periodo julio a diciembre del 2011. Tesis para obtener el título de médico. Universidad Técnica Particular de Loja, Loja.
Sagarra, R., Costa, B., Cabré, J. J., SoláMorales, O. \& Barrio, F. (2014). Costeefectividad de la intervención sobre el estilo de vida para prevenir la diabetes tipo 2. Revista Clínica Española, 214(2), 59-68.

\section{Correo electrónico:}

solellaes@yahoo.es

Revisión de pares:

Recibido:

Aceptado: 